\title{
BMJ Open Characteristics of COVID-19 clinical trials registered with ClinicalTrials.gov: cross-sectional analysis
}

\author{
Christopher W Jones (D) , ${ }^{1}$ Ashley L Woodford, ${ }^{1}$ Timothy F Platts-Mills ${ }^{2}$
}

To cite: Jones CW, Woodford AL, Platts-Mills TF. Characteristics of COVID-19 clinical trials registered with ClinicalTrials.gov: crosssectional analysis. BMJ Open 2020;10:e041276. doi:10.1136/ bmjopen-2020-041276

- Prepublication history for this paper is available online. To view these files, please visit the journal online (http://dx.doi org/10.1136/bmjopen-2020041276).

Received 03 June 2020 Revised 19 August 2020 Accepted 09 September 2020

A) Check for updates

(c) Author(s) (or their employer(s)) 2020. Re-use permitted under CC BY-NC. No commercial re-use. See rights and permissions. Published by BMJ.

${ }^{1}$ Emergency Medicine, Cooper Medical School of Rowan University, Camden, New Jersey, USA

${ }^{2}$ Emergency Medicine, University of North Carolina, Chapel Hill, North Carolina, USA

Correspondence to Dr Christopher W Jones; jones-christopher@ cooperhealth.edu

\section{ABSTRACT}

Objectives To characterise current COVID-19-related research activities.

Design Cross-sectional analysis.

Setting Clinical trials registered with ClinicalTrials.gov testing interventions relevant to COVID-19.

Data sources ClinicalTrials.gov was searched for COVID-19 and related terms to identify trials registered between 1 December 2019 and 1 May 2020 that test interventions related to the COVID-19 pandemic.

Main outcome measures We classified trials according to intervention type, and report key trial characteristics including recruitment status, location, funder type, target enrolment number, intervention model (single group, randomised or sequential assignment) and projected completion date.

Results of the 630 identified clinical trials related to COVID-19, 509 (81\%) involved the study of drugs or biological agents. Of these trials of drugs and biologics, $305(60 \%)$ use an open-label design, $43(8 \%)$ are single blinded (participant only) and 161 (32\%) are double blinded (participant and investigator). 94 (18\%) of the drug/biological trials are non-randomised. Either hydroxychloroquine or chloroquine is administered as part of the study protocol in $152(30 \%)$ of the drug/ biological trials. The total planned enrolment for these hydroxychloroquine/chloroquine trials is over 200000 participants, which represents $65 \%$ of the total planned enrolment for all registered trials of drugs or biologics. There are also at least 25 registered trials of azithromycin $(n=53)$, convalescent plasma $(n=38)$, lopinavir/ritonavir $(n=30)$, stem cell treatments $(n=29)$ and tocilizumab $(n=25) .142$ trials were registered in the first 3 months of 2020 , and 488 trials were registered between 1 April and 1 May 2020.

Conclusions These findings demonstrate a robust research response to the COVID-19 pandemic, though many of the currently planned and ongoing trials focus on a small number of potential therapies, and many also lack essential design features and power necessary to provide accurate treatment effect estimates.

\section{INTRODUCTION}

The COVID-19 pandemic has generated an unprecedented clinical research response. While this response has tremendous potential to save lives and improve the well-being of people across the globe, the magnitude of
Strengths and limitations of this study

- The study includes data from studies registered at ClinicalTrials.gov, the largest clinical trial registry in the world.

- The comprehensive search strategy used by investigators is likely to have identified most relevant trials within the ClinicalTrials.gov database.

- Registry data are subject to change over time, including estimates of the number of planned trials and the number of expected trial participants.

the response poses risks. In particular, there is a risk that the poor choice of research questions, use of suboptimal study designs and the failure to coordinate research activities will result in wasted research resources. $^{12}$ These at-risk resources include money and supplies of drugs, effort on the part of investigators and study personnel and, perhaps most importantly, time. A number of experts have predicted that even after the present COVID-19 outbreak declines, recurrent outbreaks are possible or even likely. ${ }^{3}$ It is therefore critical to make efficient use of time in the search for effective treatment and preventative measures.

Optimising the efficiency of research efforts requires some degree of coordination across the biomedical research community. A prerequisite to the coordination of COVID-19 research effort is the characterisation of current research activities. Specifically, a better understanding is needed of the types of trials planned or in progress, the specific treatments to be studied and the number of patients expected to be included. Collectively, this information has the potential to maximise the clinical utility of ongoing and future research efforts. ${ }^{4}$ Specific uses might include determining which interventions need additional research, identifying groups of studies for which harmonisation of inclusion criteria, dosing and outcomes might be beneficial to facilitate pooled analyses and 
aiding clinicians and policymakers in the interpretation of positive or negative findings from single studies. The objective of this study is to characterise current COVID19-related research activities with respect to the interventions under investigation and the design features of registered trials in order to identify opportunities to reduce wasted resource utilisation.

\section{METHODS}

\section{ClinicalTrials.gov}

As of May 2020, ClinicalTrials.gov contained protocol information for over 330000 studies from 210 different countries, making it by far the largest registry of clinical trials. ${ }^{5}$ Information for registered studies is generally entered into ClinicalTrials.gov by study sponsors or investigators, after which it undergoes a quality control process before study information is made publicly available. Study information can be updated over time, and the registry includes a public audit trail of changes made to previously posted entries. Any human subject clinical trial that is performed in accordance with local ethical and governmental regulations is eligible for registration with ClinicalTrials.gov. ${ }^{6}$ Registration with ClinicalTrials. gov is required for most human subject trials involving drugs or devices regulated by the US Food and Drug Administration that either are conducted in the USA, are conducted under an investigational new drug application or investigational device exemption, or involve the export of a drug or device manufactured in the USA. ${ }^{7}$

\section{Registry search}

We searched ClinicalTrials.gov on 1 May 2020 for trials registered after 1 December 2019 that tested interventions relevant to the COVID-19 pandemic. The search was performed using the following keywords in the ClinicalTrials.gov 'condition or disease' field: COVID-19 OR COVID-19 OR SARS-CoV-2 OR coronavirus OR corona virus OR 2019-nCoV OR 2019 novel coronavirus OR severe acute respiratory syndrome coronavirus 2. We reviewed the individual registry entries for each of the studies identified by this search, and excluded those that did not meet the definition of a clinical trial adopted by the WHO: any research study that prospectively assigns human participants or groups of humans to one or more health-related interventions to evaluate the effects on health outcomes. ${ }^{8}$ Based on this definition we excluded observational studies, expanded access studies and simulation or manikin-based studies. We also excluded studies withdrawn prior to initiating enrolment and studies not relevant to COVID-19. We classified the remaining trials according to intervention type: drugs/biologics, vaccines, behavioural health or mental health interventions, physiological interventions (eg, prone positioning, ventilator settings), medical devices and other interventions. Trials were included in more than one intervention category when relevant. Studies involving complementary or traditional medical interventions were included if they otherwise met the eligibility criteria described above.

\section{Classification of drug trials}

Those trials testing drugs or biologics were categorised according to the specific agents administered in each study. We grouped trials testing hydroxychloroquine or chloroquine together based on the similar structure and function of these drugs. ${ }^{9}$ We considered trials to involve a particular drug or biologic if that specific drug/biologic was administered to participants in any of the trial's treatment arms as a part of the trial protocol. For trials with one treatment arm we considered a drug to be an intervention of interest if it was administered to all of the subjects in that study arm; for example, we did not consider hydroxychloroquine to be an intervention of interest if participants in a single-arm trial were permitted, but not required, to receive the drug. For trials with multiple treatment arms we considered a drug to be an intervention of interest if the study design facilitated an evaluation of the drug's efficacy by either administering it to participants in some but not all of the study arms, or by administering different doses or methods of administration to participants in different arms. We also distinguished between drug/biological trials testing interventions for use in the treatment of patients with active COVID-19 disease from those testing interventions in a prophylactic capacity in order to prevent infection with COVID-19.

\section{Data collection and reporting}

We report key study design characteristics based on the registered information for each included trial. These characteristics include trial location(s), funder type(s), target enrolment number, intervention model (single group, randomised or sequential assignment) and anticipated primary completion date, which represents the date on which data collection is completed for the trial's primary outcome measure. Trials were classified as unblinded if participants were not masked to their assigned treatment group, single blind if participants were masked to the group assignment and investigators were not, and double blind if both participants and investigators were masked to the group assignment. We also describe the subject recruitment status recorded as of 1 May 2020 for each trial. This reflects the recruitment status at the time of initial registration, or as of the most recent update to the registry record for entries that have been updated. Because the recruitment status field may not be updated immediately after the initiation of enrolment, we also report the number of trials for which the registered 'Study Start Date' has passed. We also report the primary completion date for the included trials, which reflects the final date on which primary outcome data are generated for the final trial participant. We obtained counts for the total number of individuals worldwide confirmed to have COVID-19 from the WHO Coronavirus Disease Dashboard (https://COVID-19.who.int/). ${ }^{10}$ 


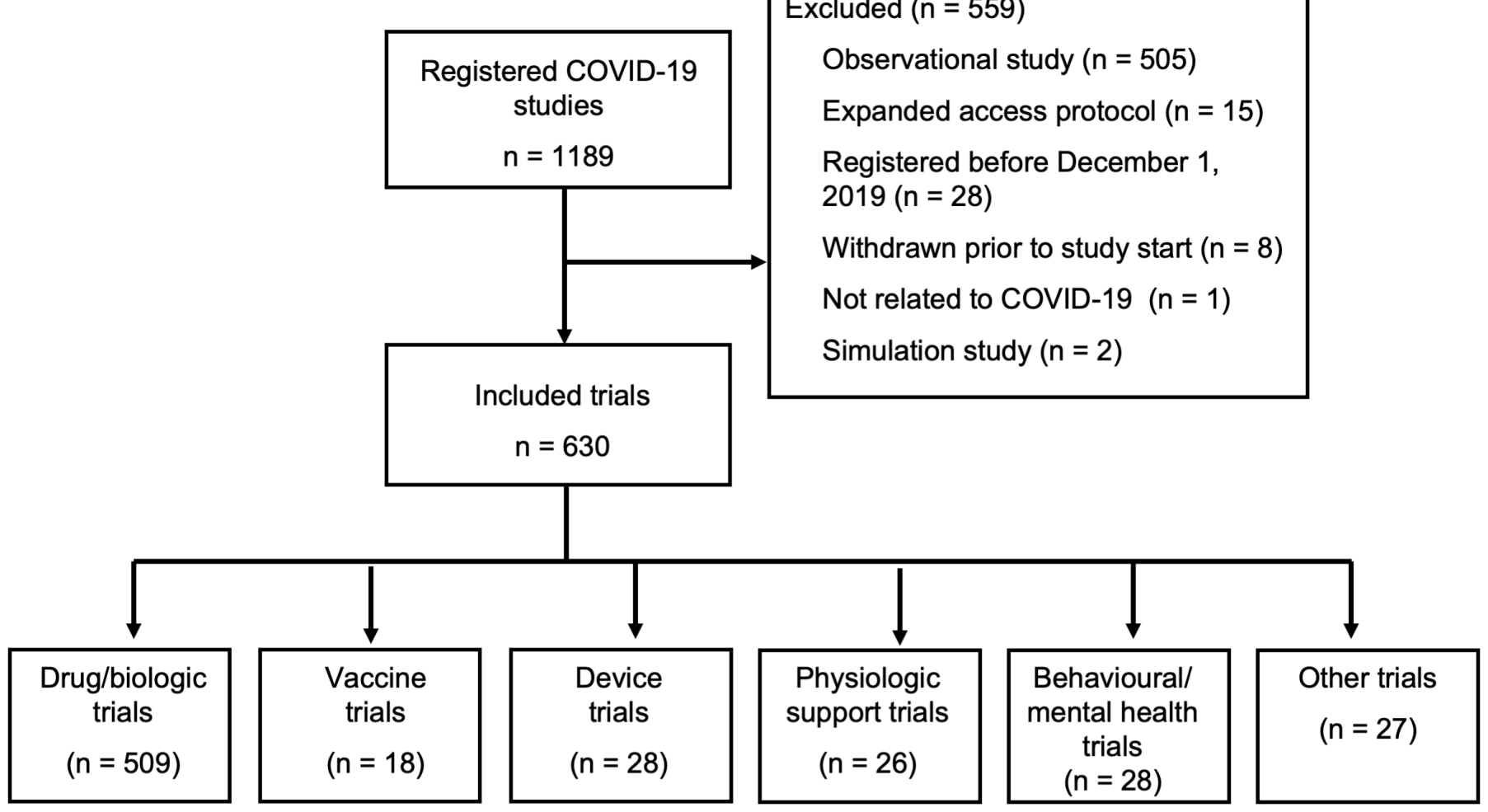

Figure 1 Flow chart of included trials.

\section{Data analysis}

We classified the included trials based on the category of intervention tested, and further classified trials of drugs or biologics based on the specific agent under investigation. Descriptive data are presented for each category, including proportions for categorical data and medians with IQRs for continuous data. When relevant the number of registry entries with missing data is reported for each categorical variable. We compared proportions using $\chi^{2}$ tests, and $p$ values $<0.05$ were considered significant. These analyses were performed using SPSS V.26.0 (SPSS).

\section{Patient and public involvement}

Patients or the public were not involved in the design, conduct, reporting or dissemination plans of this work.

\section{RESULTS}

Our search of ClinicalTrials.gov resulted in 1189 potentially eligible studies, 630 of which were clinical trials meeting our inclusion criteria (figure 1). The majority of trials $(\mathrm{n}=509,81 \%)$ are study drugs or biological agents (table 1). Twenty-six trials study physiological interventions, most of which involve the study of mechanical ventilation strategies or prone positioning. There were 18 registered trials involving vaccines; seven of these involve testing the effects of tuberculosis or measles vaccines on COVID-19 susceptibility, and 11 test vaccines specifically designed for the SARS-CoV-2 virus.

\section{Trial design characteristics}

Among the trials testing drugs or biological agents, 305 $(60 \%)$ are designed as open-label studies (table 2). Eight per cent $(n=43)$ are single blinded and less than onethird $(\mathrm{n}=161,32 \%)$ mask both participants and investigators to the group assignment. Random allocation is used in $415(82 \%)$ of the drug/biological trials; $94(18 \%)$ do not randomise participants.

\section{Prophylaxis versus treatment trials}

Among the 509 drug trials, $57(11 \%)$ test the ability of the intervention to prevent COVID-19 infection, 452 (89\%) test drugs in the context of treating active COVID-19 infections and 2 trials include both prophylaxis and treatment arms. Based on current registry data, 40 of the prophylaxis trials $(70 \%)$ involve the administration of hydroxychloroquine or chloroquine, and these 40 trials will enrol $88 \%$ of all prophylaxis trial participants. The proportion of trials using randomisation is similar between prophylaxis $(88 \%)$ and treatment trials (81\%), though prophylaxis trials are more likely to be double blinded than treatment trials ( $54 \%$ vs $29 \%, \mathrm{p}<0.001)$.

\section{Drugs under investigation}

The drug/biological trials involve the study of 218 unique agents; most of these are drugs which have been repurposed from other indications for study against COVID19 , as in the case of antimalarial treatments, antiviral agents and immunosuppressants. ${ }^{11}$ The most commonly studied drugs are hydroxychloroquine or chloroquine, 
Table 1 Characteristics of COVID-19-related clinical trials registered with ClinicalTrials.gov from 1 December 2019 to 19 April 2020

\begin{tabular}{|c|c|}
\hline Trial characteristics & $\begin{array}{l}\text { All trials } \\
(n=630)\end{array}$ \\
\hline \multicolumn{2}{|l|}{ Enrolment status, n (\%) } \\
\hline Not yet recruiting & $314(50)$ \\
\hline Recruiting & $287(46)$ \\
\hline Active, not recruiting & $13(2)$ \\
\hline Enrolment completed & $11(2)$ \\
\hline Stopped early/suspended & $5(1)$ \\
\hline \multicolumn{2}{|l|}{ Intervention, $\mathrm{n}(\%)^{\star}$} \\
\hline Drug/biologic & $509(81)$ \\
\hline Behavioural/mental health & $28(4)$ \\
\hline Physiologic & $26(4)$ \\
\hline Device & $28(4)$ \\
\hline Vaccine† & $18(3)$ \\
\hline Other & $27(4)$ \\
\hline \multicolumn{2}{|l|}{ Funding source, $\mathrm{n}(\%)^{\star}$} \\
\hline Industry & $143(23)$ \\
\hline NIH/US government & $11(2)$ \\
\hline Other/none & $558(89)$ \\
\hline \multicolumn{2}{|l|}{ Location* } \\
\hline Europe & $215(34)$ \\
\hline USA & $192(30)$ \\
\hline China & $74(12)$ \\
\hline Other Asia & $56(9)$ \\
\hline Other & $89(14)$ \\
\hline Not specified & $24(4)$ \\
\hline Number of participants, median (IQR) & $150(60-401)$ \\
\hline
\end{tabular}

*Trials may be listed in more than one category; totals therefore add to more than $100 \%$.

†Includes seven vaccine trials that study the effect of tuberculosis or measles vaccines on the incidence of COVID-19 infection rather than a vaccine specific to the novel coronavirus.

$\mathrm{NIH}$, National Institutes of Health.

which are administered in $152(30 \%)$ of the drug/ biological trials. Eighty-eight of these are treatment trials assessing hydroxychloroquine/chloroquine as a treatment of interest for patients with COVID-19, 38 study the prophylactic use of these drugs, 2 have both treatment and prophylactic arms and 24 administer hydroxychloroquine/chloroquine as part of standard treatment to all participants in multiarm studies. The total planned enrolment for the hydroxychloroquine/chloroquine trials is over 211000 participants, which represents $65 \%$ of the total planned enrolment for all currently registered trials of drugs or biologics.

Azithromycin is administered in 53 trials, 47 (89\%) of which also involve the administration of hydroxychloroquine or chloroquine. There are also 25 or more registered trials of convalescent plasma $(\mathrm{n}=38)$, lopinavir/ ritonavir $(n=30)$, stem cell treatments $(n=29)$ and tocilizumab $(n=25)$. Among these trials, the proportion with double-blinded designs ranges from $17 \%$ (lopinavir/ ritonavir) to $34 \%$ (stem cell treatments). The proportion of studies using randomised allocation ranges from $50 \%$ (convalescent plasma) to 93\% (lopinavir/ritonavir).

\section{Timing of registration and enrolment}

Based on the most recent update to the ClinicalTrials.gov 'Recruitment Status' field for each of the 630 included trials, $314(50 \%)$ had not yet started enrolment, 287 $(46 \%)$ were currently enrolling participants and 16 (3\%) had suspended or completed enrolment. As of 1 May 2020, review of each trial's registered 'Study Start Date' and 'Primary Completion Date' showed 534 trials $(85 \%)$ that had passed the date on which recruitment was scheduled to begin but had not yet reached the anticipated primary completion date. The registered primary completion date had passed for 56 trials (9\%). Analysis of the timing of registration for new interventional trials related to COVID-19 shows that 142 trials were registered in the first 3 months of 2020, and 488 trials were registered from 1 April to 1 May (figure 2). Discrepancies between data from the recruitment status field and the registered start and completion dates are likely to reflect either delays in updating the recruitment status field after this status has changed, or a change in the trial schedule relative to the schedule anticipated at the time of registration. Ninety-eight trials $(16 \%)$ are targeting completion by 31 May 2020, $244(39 \%)$ by 31 July and $346(55 \%)$ by 30 September. For 173 trials $(27 \%)$ the planned primary completion date is in 2021 or later.

\section{DISCUSSION}

As of 1 May 2020, 630 trials had been registered with ClinicalTrials.gov to study interventions related to COVID-19 infection. Together, these trials investigate 218 different drugs or biological agents, though nearly onethird involve the study of hydroxychloroquine or chloroquine. Trials of hydroxychloroquine or chloroquine plan to enrol over 210000 participants in total, which represents nearly two-thirds of the planned enrolment for all currently registered COVID-19-related trials assessing drugs or biologics. Additionally, azithromycin, convalescent plasma, lopinavir/ritonavir and stem cells are each being studied in at least 25 trials. Many of these trials lack essential design features such as double blinding (absent in 68\%) and randomised allocation of participants (absent in 18\%) that are essential for using results to accurately estimate treatment effect. ${ }^{12}$

The data included in this analysis provide an overview of many of the initial clinical trials launched in response to the COVID-19 pandemic. Several important factors should be considered when interpreting these findings. First, while ClinicalTrials.gov is the largest trial registry in the world, many trials are either only listed in other 
Table 2 Characteristics of registered COVID-19 trials assessing drugs or biological agents

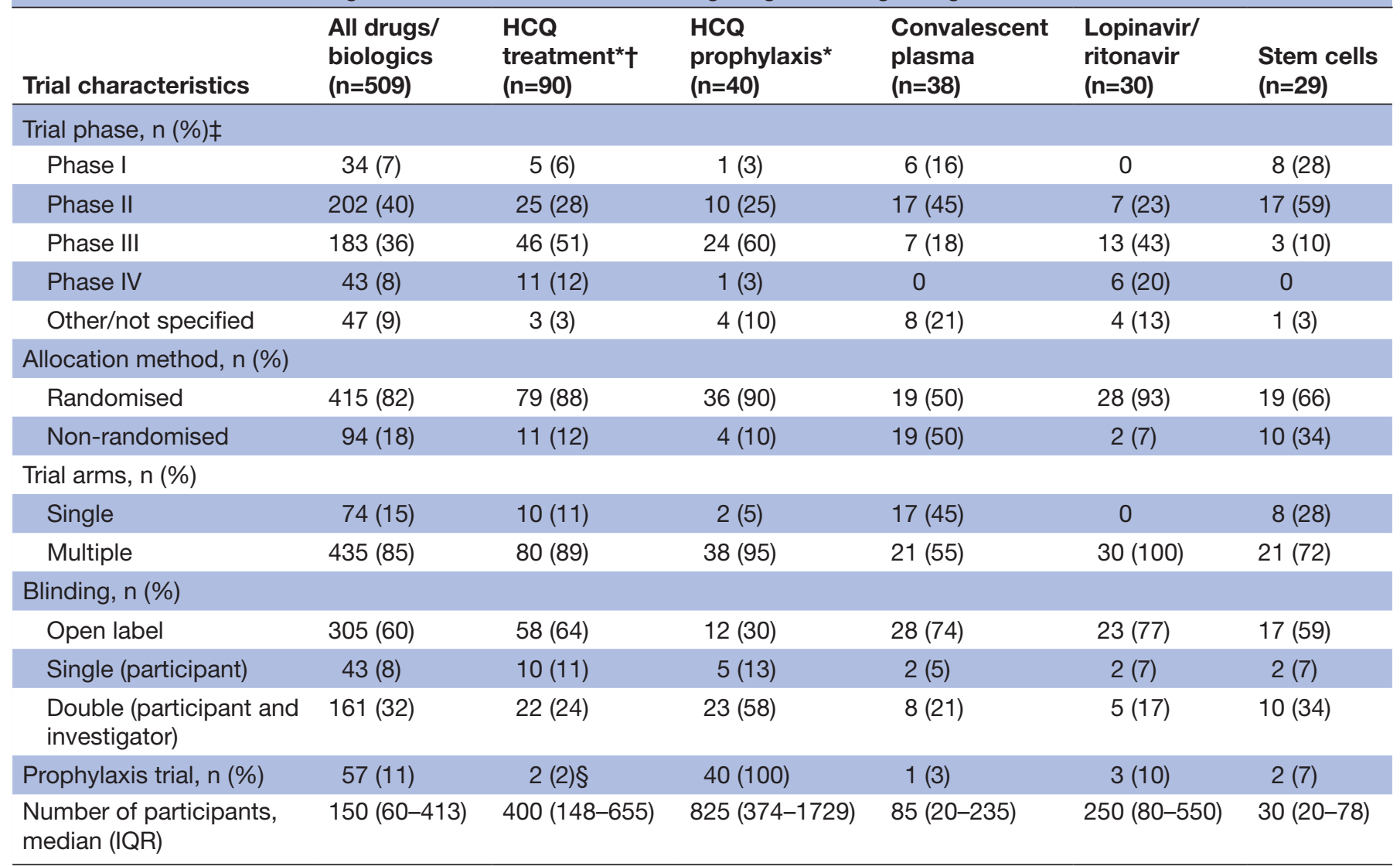

*Includes trials of hydroxychloroquine or chloroquine.

†Azithromycin trials $(n=53)$ not listed as 47 of these also involve hydroxychloroquine or chloroquine.

†Phase I/II trials classified as phase II, phase II/III trials classified as phase III.

$\S$ Two hydroxychloroquine trials included both treatment and prophylaxis arms.

$\mathrm{HCQ}$, hydroxychloroquine.

registries or not registered at all, and there may be systematic study design differences between those trials registered with ClinicalTrials.gov and those that are not. ${ }^{13-15}$ Despite this potential limitation, our data set reflects a geographically diverse cohort of trials, including a large number of trials enrolling participants in both Europe and Asia. Similarly, while we used a comprehensive search strategy to find relevant trials within the ClinicalTrials.gov database, it is possible that this search failed to identify

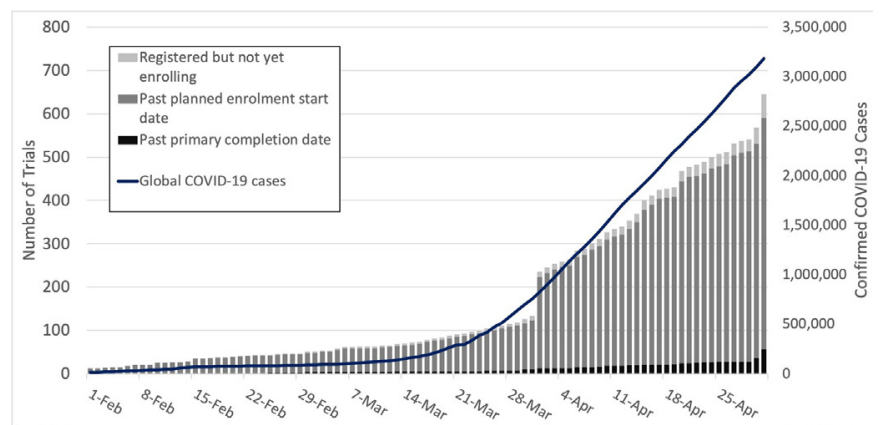

Figure 2 Timeline describing the registration and conduct of COVID-19 clinical trials along with the global total of confirmed COVID-19 cases. some relevant COVID-19 trials. Furthermore, it is also possible that some of the currently registered trials will never be initiated. Finally, we present data reflecting registry entries for each of the included trials as of 1 May 2020. While some registry data fields such as the chosen blinding strategy or the method of allocation are unlikely to change over time, others such as the recruitment status routinely change over the course of a trial. In some cases, updates to individual registry entries may be delayed, and the therefore data reflected in the public registry may be out of date. ${ }^{16}$ For example, a number of the included trials had a recruitment status indicating that enrolment had not yet started, but the registered enrolment start date had passed. These discrepancies are likely to reflect either delays in updating the recruitment status field, or changes to a trial's enrolment schedule relative to what was anticipated at the time of registration. ${ }^{17}$ Several near real-time online dashboards have been developed which provide both the research community and the public the ability to monitor characteristics, including recruiting status, of COVID-19 trials. ${ }^{18} 19$ These dashboards are valuable tools for tracking changes in the status of registered clinical trials over time, though they still rely on sponsors 
and investigators of the included trials to keep individual registry entries up to date.

Our results are consistent with other recently published studies that have characterised registered COVID-19 trials and have demonstrated that many of these trials are likely underpowered, and also lack design features such as randomised allocation and double blinding that are critical to generating bias-free estimates of treatment effects. ${ }^{2021}$ Our findings build on this work by highlighting the proportion of trials of specific interventions that fail to use these design features. Additionally, we show that current plans call for over 210000 individuals or nearly two-thirds of participants in all COVID-19 drug trials to be enrolled in trials assessing hydroxychloroquine or chloroquine. This includes $88 \%$ of all individuals enrolled in trials testing drugs for use in a preventative capacity. These findings demonstrate the substantial opportunity costs associated with a largely uncoordinated global trial response; resources spent enrolling massive numbers of participants in trials with suboptimal design features that are overwhelmingly focused on a few individual interventions like hydroxychloroquine would almost certainly be better used conducting a smaller number of high-quality trials aimed at testing other agents.

Our findings show a rapid increase in the number of trials registered starting in early April, approximately 3 weeks after the WHO declared COVID-19 a pandemic. These findings also indicate a profound lack of coordination of existing COVID-19 trials, which consequently increases the likelihood of wasted research effort and also poses risks. ${ }^{22}$ Even under normal circumstances, clinical research often results in waste due to in part inadequate power, suboptimal study design and early termination. ${ }^{21123}$ Although attempting to confirm previously observed results through the replication of prior studies is important, the simultaneous conduct of numerous trials that test the same intervention is unlikely to be an optimally efficient allocation of research resources. ${ }^{2}$ The problem of waste may be further exacerbated during the study of a pandemic disease in which (1) there exists limited relevant foundational research, (2) fluctuating case volumes make enrolment projections challenging, and (3) knowledge about the disease changes rapidly during the first few months, resulting in changes in regard to what is considered standard treatment. ${ }^{24}$ For several of the drugs studied, the conduct of numerous small trials increases the risk for spurious findings which would add to confusion regarding treatment efficacy and might make it difficult for regulatory boards and clinicians to judge if there is clinical equipoise for larger trials. ${ }^{25}$ Several of the included studies are directly competing for patients, increasing the risk that enrolment targets will not be met in a timely manner. Finally, performing pooled analyses may be challenging due to differences in inclusion criteria, dosing and outcomes ${ }^{26-28}$ Centralised coordination of studies, particularly in the form of largescale platform or adaptive trials, reduces the risk of bias and increases the potential to identify an effective treatment. ${ }^{29}{ }^{30}$ The National Institutes of Health Accelerating COVID-19 Therapeutic Interventions and Vaccines partnership is one such promising effort to improve trial coordination. ${ }^{31}$ Other important initiatives aimed at addressing these concerns through the rapid implementation of large-scale, centrally coordinated COVID-19 trials are the Solidarity Trial, sponsored by the WHO, and the Recovery Trial, sponsored by Oxford University, both of which have enrolled thousands of patients into multiarm trials testing a number of different interventions. $^{32} 33$

Further follow-up of the included trials through the results dissemination phase will ultimately help to assess the potential impact that these trials may have on clinical decision-making and patient outcomes. ${ }^{34}$ Several COVID19-related trials have already completed enrolment and results have been published. ${ }^{35}{ }^{36}$ In particular, early trial data show that remdesivir shortens the time to recovery among some hospitalised patients with COVID-19, and an open-label study of dexamethasone found evidence that steroid treatment decreases COVID-19 mortality. ${ }^{37} 38$ Results from many additional trials will be published over the coming months. Utilisation of trial registry data will be critical to the interpretation of these results by helping the medical community assess for the presence of publication bias among this initial group of publications. ${ }^{39} 40$ This will be particularly important given that there are several interventions each being studied in a large number of trials. For example, if all 88 hydroxychloroquine/chloroquine trials defined a statistically significant result based on a two-sided $p$ value of 0.05 , even in the absence of any true drug affect we would expect about four of these trials to report a statistically significant treatment effect due to chance alone. $^{25}$

Our findings illustrate the potential for ClinicalTrials. gov and other registries to aid sponsors and investigators in reducing waste by informing design decisions for future COVID-19 trials. Specifically, in addition to using study design features that help to produce unbiased estimates of treatment effects, key stakeholders should also use registry data to prioritise the conduct of trials that address important but relatively understudied clinical questions. Additionally, there is evidence that outcome data are not available for a large number of previously completed trials using drugs which are now under investigation for use in the treatment of COVID-19. ${ }^{41}$ Given the potential relevance of patient safety information collected during the course of these prior trials to the treatment of patients with COVID-19, regulators should work to secure public access to these trial data sets as a rapid and inexpensive way of using trial registries to inform both COVID-19 research priorities and patient care decisions. Future assessment of COVID-19 trials including monitoring of enrolment rates, determining characteristics associated with early termination versus successful trial completion and evaluating timely outcome reporting will help inform policies aimed at increasing the value of trials for this and subsequent pandemic events. 
Contributors CWJ conceived the study. CWJ, ALW and TFPM designed the study. CWJ and ALW performed data collection. CWJ performed the data analysis and drafted the initial manuscript. All authors contributed to revisions of the manuscript. CWJ is the guarantor of the study.

Funding The authors have not declared a specific grant for this research from any funding agency in the public, commercial or not-for-profit sectors.

Competing interests None declared.

Patient consent for publication Not required.

Provenance and peer review Not commissioned; externally peer reviewed.

Data availability statement Data are available upon reasonable request. The data set is available from the corresponding author at: jones-christopher@cooperhealth. edu on reasonable request.

Open access This is an open access article distributed in accordance with the Creative Commons Attribution Non Commercial (CC BY-NC 4.0) license, which permits others to distribute, remix, adapt, build upon this work non-commercially, and license their derivative works on different terms, provided the original work is properly cited, appropriate credit is given, any changes made indicated, and the use is non-commercial. See: http://creativecommons.org/licenses/by-nc/4.0/.

\section{ORCID iD}

Christopher W Jones http://orcid.org/0000-0001-9704-9094

\section{REFERENCES}

1 Altman DG. The scandal of poor medical research. BMJ 1994;308:283-4

2 loannidis JPA, Greenland S, Hlatky MA, et al. Increasing value and reducing waste in research design, conduct, and analysis. Lancet 2014;383:166-75.

3 Chavez C. Another wave of coronavirus will likely hit the US in the fall. Here's why and what we can do to stop it, 2020. Available: https://www.cnn.com/2020/05/02/health/coronavirus-second-wavefall-season/index.htm

4 Sanders JM, Monogue ML, Jodlowski TZ, et al. Pharmacologic treatments for coronavirus disease 2019 (COVID-19). JAMA 2020.

5 Clinicaltrials.gov: Trends, charts, and maps: U.S. National library of medicine. Available: https://clinicaltrials.gov/ct2/resources/trends [Accessed 11 May 2020].

6 Clinicaltrials.gov: Submit studies: U.S. National library of medicine. Available: https://clinicaltrials.gov/ct2/manage-recs [Accessed 11 May 2020].

7 National Institutes of Health, Department of Health and Human Services. Clinical trials registration and results information submission. final rule. Fed Regist 2016;81:64981-5157.

$8 \mathrm{WHO}$. International clinical trials registry platform (ICTRP). Available: https://www.who.int/ictrp/en/ [Accessed 11 May 2020].

9 Ferner RE, Aronson JK. Chloroquine and hydroxychloroquine in covid-19. BMJ 2020;369:m1432.

10 WHO. WHO coronavirus disease (COVID-19) Dashboard, 2020. Available: https://covid19.who.int/ [Accessed 9 May 2020].

11 Lythgoe MP, Middleton P. Ongoing clinical trials for the management of the COVID-19 pandemic. Trends Pharmacol Sci 2020;41:363-82.

12 Savović J, Jones HE, Altman DG, et al. Influence of reported study design characteristics on intervention effect estimates from randomized, controlled trials. Ann Intern Med 2012;157:429-38.

13 Hunter KE, Seidler AL, Askie LM. Prospective registration trends, reasons for retrospective registration and mechanisms to increase prospective registration compliance: descriptive analysis and survey. BMJ Open 2018;8:e019983.

14 Loder E, Loder S, Cook S. Characteristics and publication fate of unregistered and retrospectively registered clinical trials submitted to The BMJ over 4 years. BMJ Open 2018;8:e020037.

15 Tan AC, Jiang I, Askie L, et al. Prevalence of trial registration varies by study characteristics and risk of bias. J Clin Epidemiol 2019;113:64-74.

16 Tse T, Fain KM, Zarin DA. How to avoid common problems when using ClinicalTrials.gov in research: 10 issues to consider. BMJ 2018;361:k1452
17 Jones CW, Safferman MR, Adams AC, et al. Discrepancies between ClinicalTrials.gov recruitment status and actual trial status: a crosssectional analysis. BMJ Open 2017;7:e017719.

18 Evidence-Based medicine Datalab at the University of Oxford. COVID-19 trials Tracker. Available: http://covid19.trialstracker.net [Accessed 18 Aug 2020].

19 Cytel. Global coronavirus COVID-19 clinical trial Tracker. Available: https://www.covid-trials.org [Accessed 18 Aug 2020].

20 Mehta HB, Ehrhardt S, Moore TJ, et al. Characteristics of registered clinical trials assessing treatments for COVID-19: a cross-sectional analysis. BMJ Open 2020;10:e039978.

21 Huang J, He Y, Su Q, et al. Characteristics of COVID-19 clinical trials in China based on the registration data on ChiCTR and ClinicalTrials. gov. Drug Des Devel Ther 2020;14:2159-64.

22 Chalmers I, Bracken MB, Djulbegovic B, et al. How to increase value and reduce waste when research priorities are set. Lancet 2014:383:156-65.

23 Ioannidis JPA. Clinical trials: what a waste. BMJ 2014;349:97089.

24 Klitzman R. Evolving challenges and Research-Needs concerning Ebola. Am J Public Health 2015;105:1513-5.

25 Li G, Taljaard M, Van den Heuvel ER, et al. An introduction to multiplicity issues in clinical trials: the what, why, when and how. Int $J$ Epidemiol 2017;46:746-55.

26 Bangdiwala SI, Bhargava A, O'Connor DP, et al. Statistical methodologies to pool across multiple intervention studies. Trans/ Behav Med 2016;6:228-35.

27 da Costa BR, Juni P. Systematic reviews and meta-analyses of randomized trials: principles and pitfalls. Eur Heart $J$ 2014;35:3336-45.

28 Halpern SD, Karlawish JHT, Berlin JA. The continuing unethical conduct of underpowered clinical trials. JAMA 2002;288:358-62.

29 Adaptive Platform Trials Coalition. Adaptive platform trials: definition, design, conduct and reporting considerations. Nat Rev Drug Discov 2019;18:797-807.

30 Berry SM, Connor JT, Lewis RJ. The platform trial: an efficient strategy for evaluating multiple treatments. JAMA 2015;313:1619-20.

31 National Institutes of Health. NIH to launch public-private partnership to speed COVID-19 vaccine and treatment options. Available: https:// www.nih.gov/news-events/news-releases/nih-launch-public-privatepartnership-speed-covid-19-vaccine-treatment-options [Accessed 26 Apr 2020].

32 ISRCTN Registry. Public health emergency solidarity trial of treatments for COVID-19 infection in hospitalized patients. Available: http://www.isrctn.com/ISRCTN83971151 [Accessed 16 Aug 2020].

33 ClinicalTrials.gov. Randomised evaluation of COVID-19 therapy (recovery). Available: https://clinicaltrials.gov/ct2/show/ NCT04381936

34 Glasziou P, Altman DG, Bossuyt P, et al. Reducing waste from incomplete or unusable reports of biomedical research. Lancet 2014;383:267-76.

35 Borba MGS, Val FFA, Sampaio VS, et al. Effect of high vs low doses of chloroquine diphosphate as adjunctive therapy for patients hospitalized with severe acute respiratory syndrome coronavirus 2 (SARS-CoV-2) infection. JAMA Netw Open 2020;3:e208857.

36 Cao B, Wang Y, Wen D, et al. A trial of Lopinavir-Ritonavir in adults hospitalized with severe Covid-19. N Engl J Med 2020;382:1787-99.

37 Beigel JH, Tomashek KM, Dodd LE, et al. Remdesivir for the treatment of Covid-19 - preliminary report. N Engl J Med Overseas Ed 2020:NEJMoa2007764.

38 RECOVERY Collaborative Group, Horby P, Lim WS, et al. Dexamethasone in Hospitalized Patients with Covid-19 - Preliminary Report. N Engl J Med 2020. doi:10.1056/NEJMoa2021436. [Epub ahead of print: 17 Jul 2020].

39 Song SY, Koo D-H, Jung S-Y, et al. The significance of the trial outcome was associated with publication rate and time to publication. J Clin Epidemiol 2017;84:78-84.

40 DeVito NJ, Goldacre B. Catalogue of bias: publication bias. BMJ Evid Based Med 2019;24:53-4.

41 Rodgers F, Pepperrell T, Keestra S, et al. Missing clinical trial data: the knowledge gap in the safety of potential COVID-19 drugs. medRxiv 2020. 